\title{
Propuestas para Medir la Velocidad de la Luz en Diferentes Medios dentro de un Ámbito
} Docente

\author{
José-Víctor Rodríguez ${ }^{\# 1}$, Ignacio Rodríguez-Rodríguez ${ }^{* 2}$ y José Luis Serrano Martínez ${ }^{\# 3}$ \\ ${ }^{\# 1}$ Departamento de Tecnologías de la Información y las Comunicaciones. Universidad Politécnica de Cartagena \\ Antiguo Cuartel de Antigones. Plaza del Hospital, 1, 30202. Cartagena, Murcia. España \\ 1 jvictor.rodriguez@upct.es \\ ${ }^{* 2}$ Departamento de Ingeniería de la Información y las Comunicaciones. Universidad de Murcia \\ Campus de Espinardo, 30100. Murcia. España \\ 2 ignacio.rodriguez1@um.es \\ ${ }^{\# 3}$ Departamento de Ingeniería Minera, Geológica y Cartográfica. Universidad Politécnica de Cartagena. Antiguo Hospital \\ de Marina. Plaza del Hospital, 1. 30202, Cartagena, Murcia, España. \\ 3 jose.serrano@upct.es \\ Recibido: 09/11/17; Aceptado: 28/11/17
}

Resumen - En este trabajo, se proponen una serie de experimentos, relativamente sencillos, destinados a medir la velocidad de la luz, en diferentes medios, dentro de un ámbito docente. De este modo, se describen, paso a paso, los diversos métodos y procedimientos que pueden llevarse a cabo, en un entorno de laboratorio universitario, para medir con qué velocidad se propaga la luz cuando ésta viaja por el aire, cristal o agua. Las actividades de medición de la velocidad de la luz presentadas en este artículo suponen una manera divertida y práctica de enriquecer la enseñanza de la óptica y el electromagnetismo, familiarizarse con instrumentación específica como los osciloscopios o los emisores/receptores ópticos o entender la ciencia subyacente tras las fibras ópticas. Finalmente, los experimentos propuestos representan, al fin y al cabo, un ejemplo ideal para acercar a los estudiantes el llamado "método científico".

\begin{abstract}
In this work, a series of relatively simple experiments aimed at measuring the speed of light in different media within an educational environment is presented. In this way, the different methods and procedures -which can be carried out within university laboratories- for measuring at what velocity light propagates through air, crystal or water are described step by step. The speed of light measurement activities presented in this article represent an enjoyable and practical way of enriching the teaching of both optics and electromagnetism, get familiar with specific instrumentation such as oscilloscopes and optical transmitters/receivers or understand the science underlying fiber optics. Finally, the proposed experiments represent, in the end, an ideal example of bringing students closer to the so-called 'scientific method'.
\end{abstract}

\section{INTRODUCCIÓN}

Existen en la literatura algunos artículos en los que se proponen diversos experimentos encaminados a medir la velocidad de la luz dentro de un ámbito docente, es decir, con el ánimo de llevar a cabo una experiencia didáctica en la que se utilicen medios tecnológicos que puedan encontrarse en un laboratorio universitario. De esta forma, se permite que los estudiantes conozcan o afiancen, de una manera práctica, conceptos de óptica o electromagnetismo y, además, se consigue que se familiaricen con instrumentación específica como los osciloscopios o los transmisores/receptores ópticos. Al fin y al cabo, estos experimentos potencian el desarrollo de la llamada "competencia científica" en los estudiantes a través de su vertiente investigadora, ya que, tal y como apuntaron Pilot y Bulte, ésta debe enmarcarse en contextos docentes en los que los alumnos puedan tener acceso a la realización de experimentos, con equipamiento específico, que posibilite el aprendizaje de conceptos a través de su aplicación a la vida real [1][2]. De esta manera, no sólo se favorece la adquisición de conocimiento y el aprendizaje de las ciencias, sino que se propicia la aparición de nuevas vocaciones científicas [3].

La mayoría de los artículos que han sido publicados en la literatura relativos a la medida de la velocidad de la luz dentro de un marco docente lo hacen considerando el aire como medio de transmisión y asumiendo alguna variación del método que consiste básicamente en medir el tiempo exacto que emplea un pulso de luz en recorrer una distancia conocida en relación a otro que no la recorre. De esta forma, dividiendo la distancia entre el tiempo empleado, se obtiene la velocidad de la luz buscada. Ejemplos de estos artículos son los siguientes. En el de Biretta y Lang, se hace uso de un mecanismo de rotación de espejos para diferenciar los dos pulsos de luz, de manera que ambos recorran caminos diferentes hasta ser detectados [4]. En [5], sin embargo, se hace uso de dos telescopios newtonianos para la 
gestión de los pulsos de luz. Por otra parte, el método ofrecido por Tyler se caracteriza principalmente por introducir un LED para la generación de los pulsos de luz en lugar de un láser [6]. La misma metodología se puede encontrar también en [7]. Sin embargo, en [8] realizan la medición del tiempo a partir de los ceros de la transformada de Fourier de los pulsos de luz, ya que estos están directamente relacionados con el retardo temporal entre dichos pulsos. Ejemplos más recientes de este tipo de artículos son los que pueden encontrarse en [9] y [10], donde los pulsos de luz son generados con un puntero láser, y el de Aoki y Mitsui, que presenta como características diferenciadoras que se necesite muy poco espacio para realizar el experimento y la utilización de un láser pulsado (en vez de modulado por senos) de baja potencia, con el que se evitan posibles accidentes, se facilita la medida del tiempo de propagación de la luz y se incrementa la robustez frente a distorsiones [11].

Por otra parte, en relación a artículos en los que se realice un experimento docente de medida de la velocidad de la luz en un medio que no sea el aire, encontramos el de Gülmez, en el que los pulsos de luz recorren una distancia conocida a través de cristal (el interior de fibras ópticas) [12] o el de Brody et al., en el que se calcula la velocidad de la luz en el agua mediante el método de Foucault, que implica unos factores de corrección matemáticos algo complejos [13].

En lo que se refiere a la literatura en español relativa a la medida de la velocidad de la luz, tan sólo encontramos ejemplos como los mostrados en [14] y [15], donde la velocidad de la luz se calcula emulando el conocido método de Römer basado en las observaciones astronómicas de la luna de Júpiter, Ío [16], y el ingenioso y lúdico método de Toledo y López, en el que la velocidad de la luz se calcula con ayuda de un microondas y una golosina [17] pero, en cualquier caso, siempre, el medio de transmisión considerado es el aire. Sin embargo, hasta donde estos autores conocen, no existen artículos publicados en español de experimentos que consideren otros entornos de propagación de la luz que no sean el aire $\mathrm{y}$, mucho menos, que realicen una compilación de métodos de laboratorio docente de medición de la velocidad de la luz en diferentes medios.
Por tanto, en este trabajo, se presenta una serie de métodos, que pueden ser realizados con relativa facilidad en un laboratorio docente, para medir la velocidad de la luz en diferentes medios: aire -mediante un experimento basado en el que realizó Michelson [18]-, cristal (utilizando fibras ópticas) y agua - realizando un método indirecto basado en el cálculo del índice de refracción del agua-.

\section{A. Breve Historia de la Medida de la Velocidad de la Luz.}

Hasta el siglo XVII, los científicos estaban convencidos de que la luz se propagaba de manera instantánea de un sitio a otro o, dicho de otro modo, de que su velocidad era infinita. Pensadores insignes como Aristóteles (384-322 a. c.) o, con bastante más posterioridad, René Descartes (1596-1650) así lo creían. Este hecho venía, sin duda, motivado por la experiencia de increíble rapidez que nos trasmite la luz cuando ésta viaja a lo largo de las (para ella) pequeñas distancias que se pueden encontrar en nuestro planeta. Sin embargo, en 1629, Isaac Beeckman, amigo de René Descartes, fue el primero en sugerir comprobar (aunque sin éxito) la finitud de la velocidad de la luz en el aire a través de la reflexión del destello procedente del disparo de un cañón en un espejo situado a $1,6 \mathrm{~km}$. Posteriormente, Galileo Galilei, en 1638, propuso un experimento basado en la medición del tiempo transcurrido entre la emisión de una señal luminosa por parte de un experimentador y su detección visual por parte de otra persona situada a un $1 \mathrm{~km} \mathrm{[19][20].} \mathrm{De} \mathrm{esta} \mathrm{forma,} \mathrm{se} \mathrm{podía}$ obtener la velocidad de la luz como el cociente entre dicha distancia y el tiempo medido. Sin embargo, tampoco tuvo éxito, ya que ni la distancia era lo suficientemente grande ni el tiempo de respuesta de la visión humana lo suficientemente pequeño. No fue hasta 1675 que un astrónomo danés, Ole Römer, calculó, por primera vez, un valor de la velocidad de la luz a partir de un método basado en las observaciones astronómicas del movimiento de unos de los satélites de Júpiter, Ío [16]. Así, Rømer observó que los eclipses de Ío se adelantaban o se retrasaban en función de la distancia que hubiera en ese momento Tierra-Júpiter, por lo que este desfase temporal debía estar relacionado con la velocidad finita de la luz. De esta forma, su método arrojó un resultado de $2,14 \cdot 10^{8} \mathrm{~m} / \mathrm{s}$ que, si bien estuvo algo alejado del valor actualmente 
reconocido de la velocidad de la luz en el vacío, $2,997 \cdot 10^{8} \mathrm{~m} / \mathrm{s}$ [21], tuvo una precisión bastante aceptable, dados los medios de la época. Casi 200 años después, en 1849, Armand H. L. Fizeau, realizando ya un experimento de Física dentro de la Tierra, obtuvo un valor de $3,15 \cdot 10^{8} \mathrm{~m} / \mathrm{s}$ reflejando, en un espejo situado a $8 \mathrm{~km}$, un haz de luz que antes tenía que atravesar una rueda dentada cuya velocidad se iba incrementando hasta que el rayo quedara eclipsado [22]. Poco después, en 1850, Léon Foucault mejoró el experimento de Fizeau sustituyendo la rueda dentada por un espejo giratorio e incorporando una lente que facilitaba la incidencia del rayo de luz sobre el espejo [23], obteniendo un valor de $2,980 \cdot 10^{8} \mathrm{~m} / \mathrm{s}$, situado ya en los márgenes cercanos de su valor moderno. Finalmente, Albert Michelson perfeccionó, en 1879, el montaje de Foucault modificando las distancias entre la fuente de luz, la lente y el espejo giratorio con el fin de obtener un camino total recorrido por la luz más largo [18]. El valor final que obtuvo fue de $2,999 \cdot 10^{8} \mathrm{~m} / \mathrm{s}$, realmente ajustado ya al valor conocido en la actualidad. Por supuesto, con posterioridad se fueron sucediendo muchos experimentos más por parte de innumerables científicos hasta llegar al valor actual, pero se han querido resaltar los anteriores por su relevancia histórica.

\section{DESCRIPCIÓN DE LOS EXPERIMENTOS}

A. Medida de la velocidad de la luz en el aire

- Equipamiento necesario

- Osciloscopio.

- Emisor y receptor ópticos con reflector interno.

- Lente.

- Espejo.

- Cables BNC-BNC.

- Cinta métrica.

- Calculadora.

- Metodología

Para la estimación de la velocidad de la luz en el aire se propone el montaje, basado en los experimentos de Michelson, que puede apreciarse en la Fig. 1.

Dos pulsos de luz son generados, en el mismo instante de tiempo, por un emisor óptico (LED), de manera que uno de ellos es reflejado por un reflector interno al bloque emisor (es decir, no

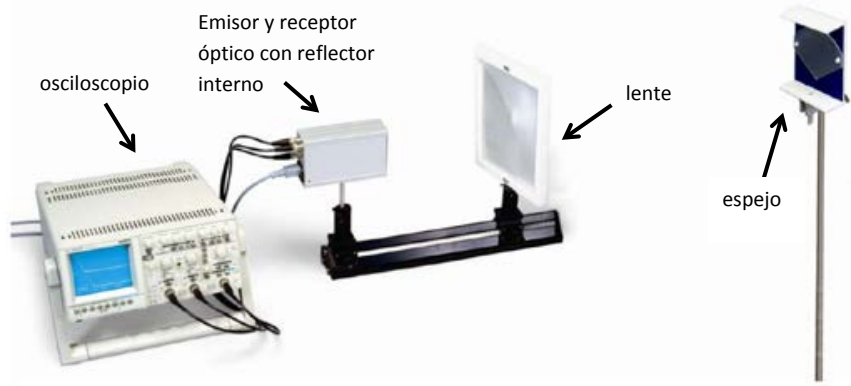

Fig. 1. Montaje para la medida de la velocidad de la luz en el aire según el experimento de Michelson. Fuente: https://www.3bscientific.es.

llega a recorrer prácticamente ningún trayecto) y el otro es lanzado fuera del emisor de forma que recorre una distancia conocida hasta impactar y reflejarse en un reflector externo (espejo), -al que converge con ayuda de una lente-, y vuelve a recorrer el mismo camino para introducirse de nuevo en el bloque inicial. Ambos pulsos de luz (el que no llega a salir del bloque emisor, denominado de referencia, y el que recorre dos veces la distancia que separa el emisor del espejo) son detectados por un receptor óptico ubicado junto al emisor y, a continuación, son visualizados en un osciloscopio. De esta forma, midiendo la diferencia de tiempos empleados en ser detectados ambos pulsos de luz (el de referencia se detectó de manera prácticamente instantánea), puede deducirse la velocidad de la luz que recorrió dos veces la distancia hasta el espejo (que es conocida), ya que, como es sabido:

$$
v=\frac{d}{t}
$$

donde $v$ es la velocidad, $d$ es la distancia recorrida y $t$ hace referencia al tiempo empleado.

- Resultados

En las Fig. 2 y Fig. 3, puede observarse el montaje llevado a cabo en el laboratorio TSC-5 de la Escuela Técnica Superior de Ingeniería de Telecomunicación (ETSIT) de la Universidad Politécnica de Cartagena (UPCT). Cabe resaltar cómo se aprecia el punto rojo en el espejo, derivado de la reflexión del pulso de luz que es lanzado fuera del emisor óptico.

Para visualizar la diferencia de tiempos de detección de los dos pulsos de luz se puede utilizar un osciloscopio Tektronic TDS 2022B, como el que se muestra en la Fig. 4, que se conecta a las diferentes salidas de la parte 


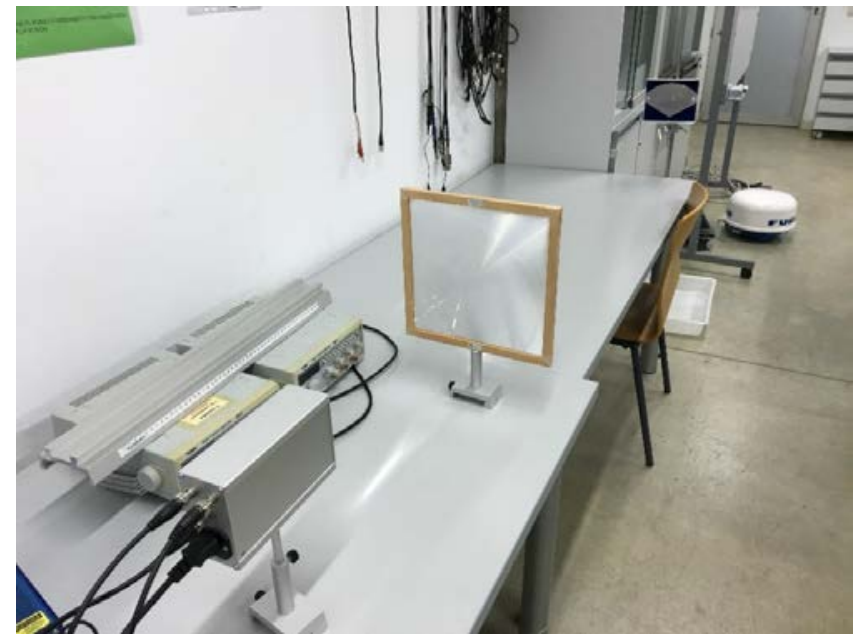

Fig. 2. Vista lateral del montaje experimental para la medida de la velocidad de la luz en el aire según el experimento de Michelson.

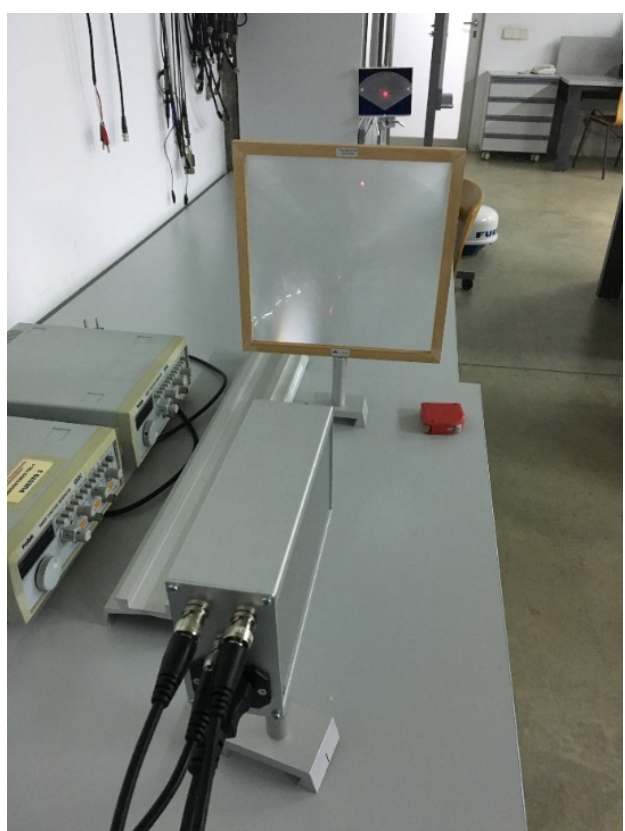

Fig. 3. Vista frontal del montaje experimental para la medida de la velocidad de la luz en el aire según el experimento de Michelson.

posterior del bloque emisor, tal y como muestra el esquema de la Fig. 5.

Como puede observarse, la salida A, que se introduce en el Canal 1 del osciloscopio (CH1), se corresponde con el pulso de luz que ha sido emitido fuera del emisor, se ha reflejado en el espejo, y ha vuelto a introducirse de nuevo en el bloque inicial (y, por tanto, ha sido detectado de manera retardada respecto al pulso de referencia).

La salida B, que se introduce en el Canal 2 del osciloscopio ( $\mathrm{CH} 2$ ), se corresponde con el pulso de luz de referencia (que no ha llegado a recorrer

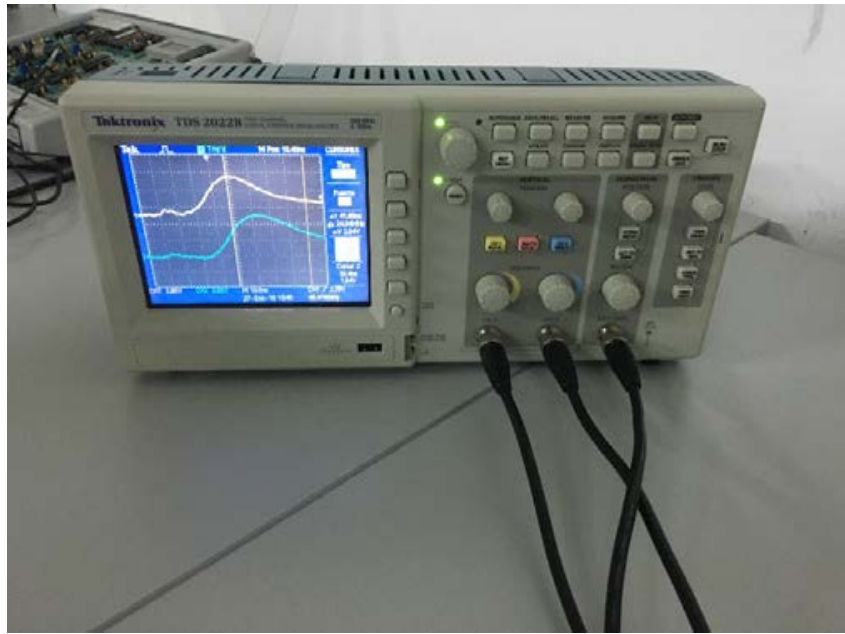

Fig. 4. Osciloscopio Tektronic TDS 2022B empleado para la visualización de la diferencia de tiempos de detección de los dos pulsos de luz emitidos.

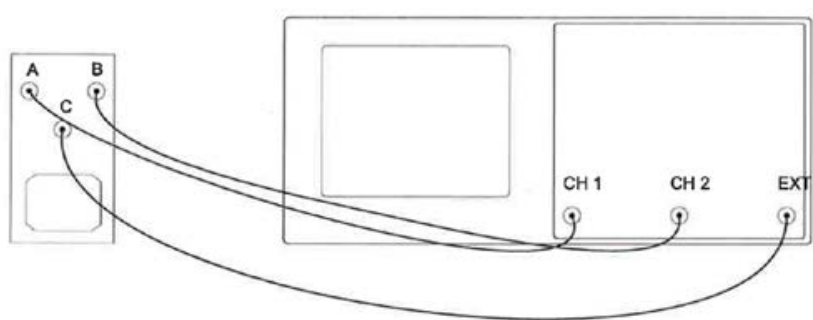

Fig. 5. Diagrama de conexión del osciloscopio a la parte posterior del bloque emisor. Fuente: https://www.3bscientific.es.

ninguna distancia) y la salida $\mathrm{C}$, que se conecta a la entrada de External Trigger del osciloscopio (EXT), proporciona un pulso de sincronización (de disparo o trigger) que tiene como objetivo sincronizar las capturas de los dos pulsos que queremos representar para que el desfase temporal que se observe entre ellos se deba exclusivamente a la diferencia de caminos recorridos, y no a un retardo generado por el propio osciloscopio. De esta manera, se visualizan los dos pulsos de luz que se aprecian en las pantallas del osciloscopio de las Fig. 6 y Fig. 7, donde, con ayuda de cursores que se sitúan en el punto de máxima amplitud de cada uno de ellos, se pueden extraer sus tiempos exactos de detección.

Concretamente, y tal y como puede apreciarse en el ejemplo de las Fig. 6 y Fig. 7, el pulso de luz de referencia se detectó en un tiempo exacto de $8,8 \mathrm{~ns}$, y el pulso de luz que se reflejó en el espejo exterior se detectó en un tiempo de 22,8 ns. 


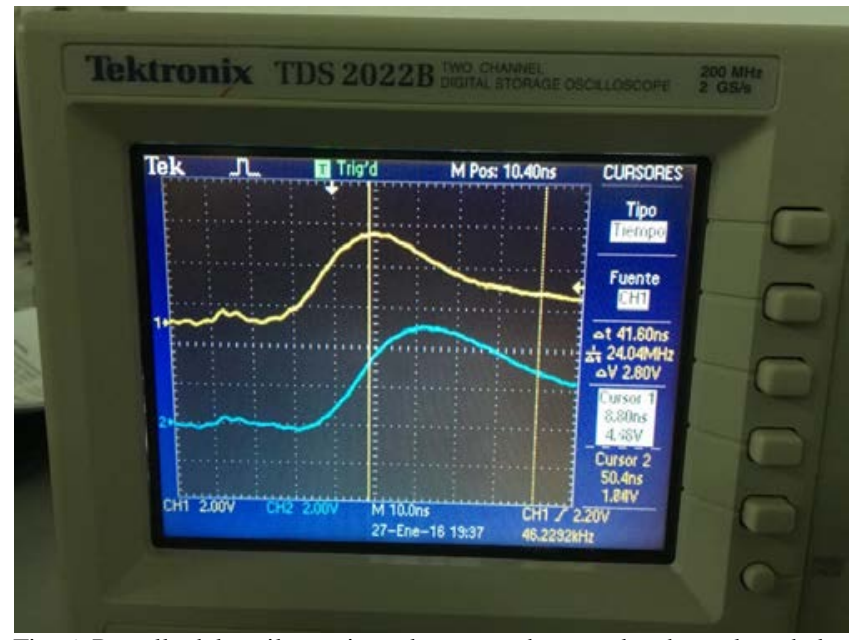

Fig. 6. Pantalla del osciloscopio en la que se observan los dos pulsos de luz emitidos, con el cursor situado en el punto de máxima amplitud del de referencia.

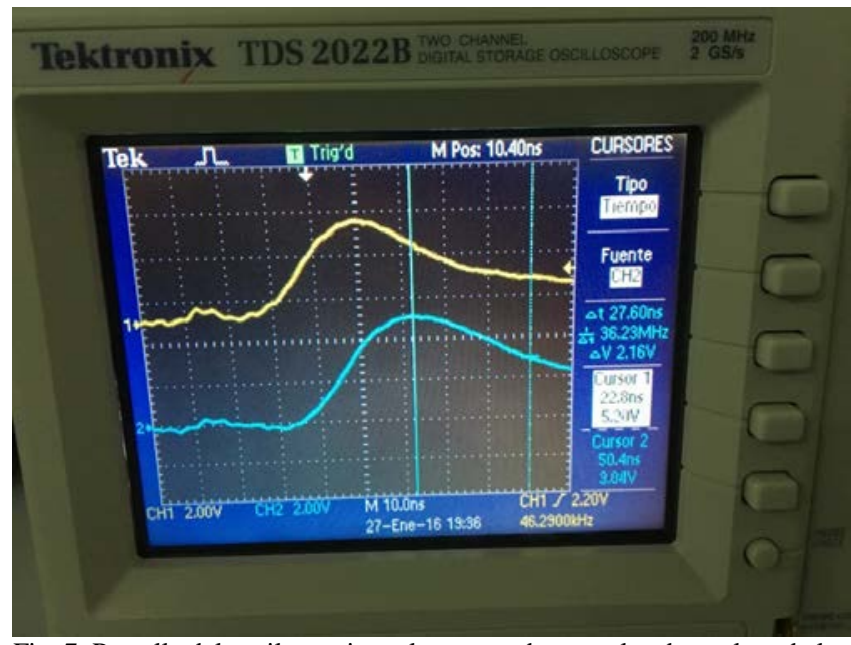

Fig. 7. Pantalla del osciloscopio en la que se observan los dos pulsos de luz emitidos, con el cursor situado en el punto de máxima amplitud de reflejado en el espejo externo.

Como se asume que el pulso de referencia no recorre ninguna distancia, la diferencia temporal en la detección de ambos pulsos fue causada por el tiempo que tardó el pulso que salió fuera del bloque emisor en recorrer dos veces la distancia entre este bloque y el espejo.

Por otro lado, la distancia entre el emisor y el espejo $(a+b$ en la Fig. 8) fue medida de manera precisa y arrojó un valor de 2,16 m.

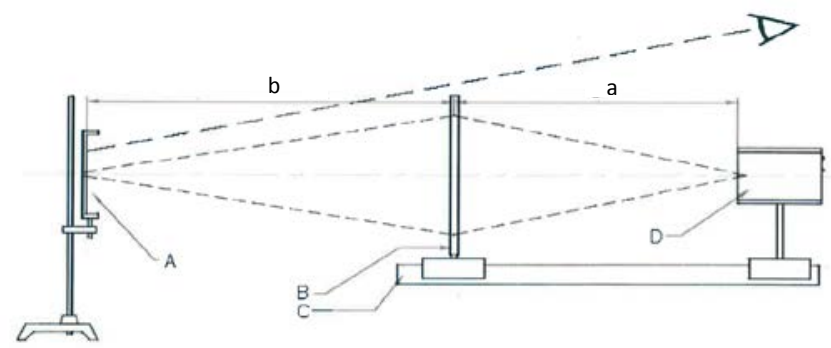

$$
\begin{gathered}
v_{\text {aire }}=\frac{2,16 \cdot 2}{22,8 \cdot 10^{-9}-8,8 \cdot 10^{-9}}= \\
\frac{4,32}{14 \cdot 10^{-9}}=3,085 \cdot 10^{8} \mathrm{~m} / \mathrm{s}=308571 \mathrm{~km} / \mathrm{s}
\end{gathered}
$$

Se observa cómo se aproxima bastante al valor de la velocidad de la luz en el aire conocido (299705 km/s). Concretamente, se ha obtenido con un error del 2,95\%.

\section{B. Medida de la velocidad de la luz en cristal}

En este caso, la medida de la velocidad de la luz en cristal se llevará a cabo mediante un montaje con fibras ópticas.

\section{- Equipamiento necesario}

- Osciloscopio.

- Placa con emisor LED y receptor óptico.

- Fibras ópticas de 20 m y 15 cm.

- Sondas BNC-cocodrilo.

- Calculadora.

- Fibras Ópticas

Las fibras ópticas (Fig. 9) son un medio de transmisión de datos, empleado en el ámbito de las telecomunicaciones, que es capaz de enviar una gran cantidad de información, hasta distancias muy elevadas, a través de pulsos de luz que son guiados dentro de su delgada estructura cilíndrica transparente (cristal de silicio). De esta manera, las fibras representan uno de los mayores avances -en lo que a transmisión de señales se refiereque se han realizado en los últimos años, además de ofrecer toda una serie de aplicaciones alternativas que comprenden, entre otras, su utilización en el ámbito médico, industrial o decorativo.

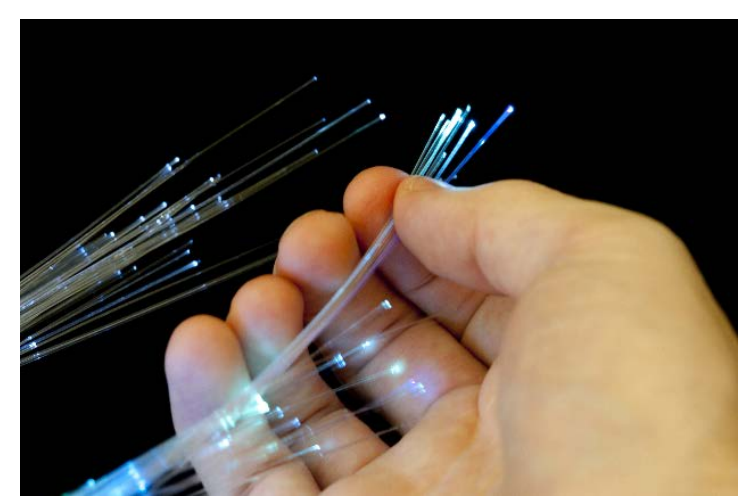

Fig. 9. Fibras ópticas transmitiendo información a través de la luz guiada en su interior. Fuente: http://sciencestockphotos.com. 
- Índice de refracción

El índice de refracción de un medio (n) es una medida para saber cuánto se reduce la velocidad de la luz (considerada como referencia la del vacío) dentro de ese medio. De esta manera, la velocidad de la luz en cualquier medio puede calcularse como:

$$
v_{\text {medio }}=\frac{c}{n}
$$

donde $c$ es la velocidad de la luz en el aire y $n$ indica el índice de refracción del medio.

El índice de refracción en el vacío es 1 y, en el aire, es de 1,00029, por lo que, muchas veces, se suele asumir que el del aire también es 1.

\section{- Metodología}

En este caso, se estimó la velocidad de la luz a través de cristal por medio de un montaje en el que, transmitiendo un pulso de luz, emitido por un LED, a través de una fibra óptica de 20 m (cuyo interior es de cristal de silicio) hasta un receptor (Fig. 10), se establece, en el osciloscopio, el tiempo empleado por dicho pulso en recorrer el tramo de fibra en cuestión (Fig. 11). De este modo, la velocidad de la luz en el cristal puede calcularse con la expresión:

$$
v_{\text {cristal }}=\frac{d_{\text {fibra }}}{t}
$$

donde $d_{\text {fibra }}$ es la distancia recorrida (longitud de la fibra) y $t$ es el tiempo empleado.

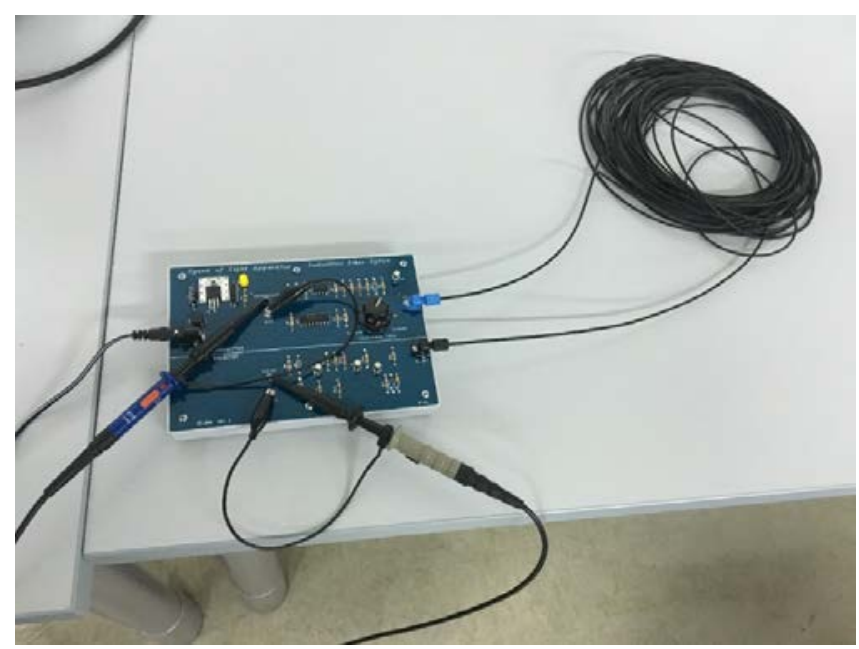

Fig. 10. Propagación de un pulso de luz a través de la fibra óptica de $20 \mathrm{~m}$.

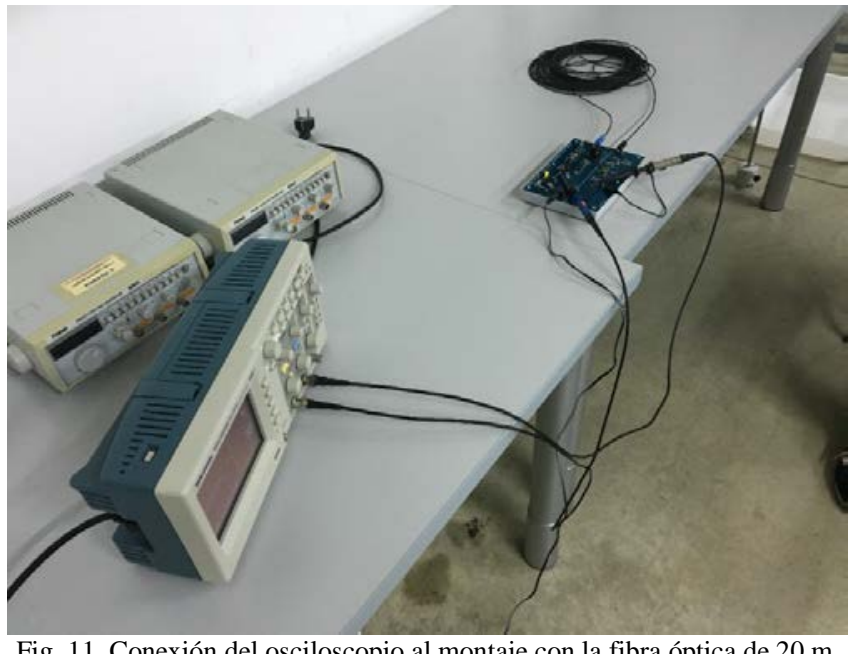

Fig. 11. Conexión del osciloscopio al montaje con la fibra óptica de 20 m.

\section{- Resultados}

Primeramente, se calibró el montaje por medio de un pequeño tramo de fibra óptica de $15 \mathrm{~cm}$ (de longitud que podemos considerar despreciable, ya que genera un retardo entre el pulso de luz emitido $y$ el recibido de menos de un nanosegundo). Esta calibración pretende eliminar los retardos en la propagación de la luz que pudieran estar causados por la propia electrónica de la placa utilizada (Fig. 12). De esta manera, se asegura que cuando, posteriormente, se coloque, entre el emisor y el receptor, el tramo de fibra de $20 \mathrm{~m}$, podamos estar seguros de que el retardo entre el pulso emitido y el recibido se debe exclusivamente a la distancia recorrida por la luz.

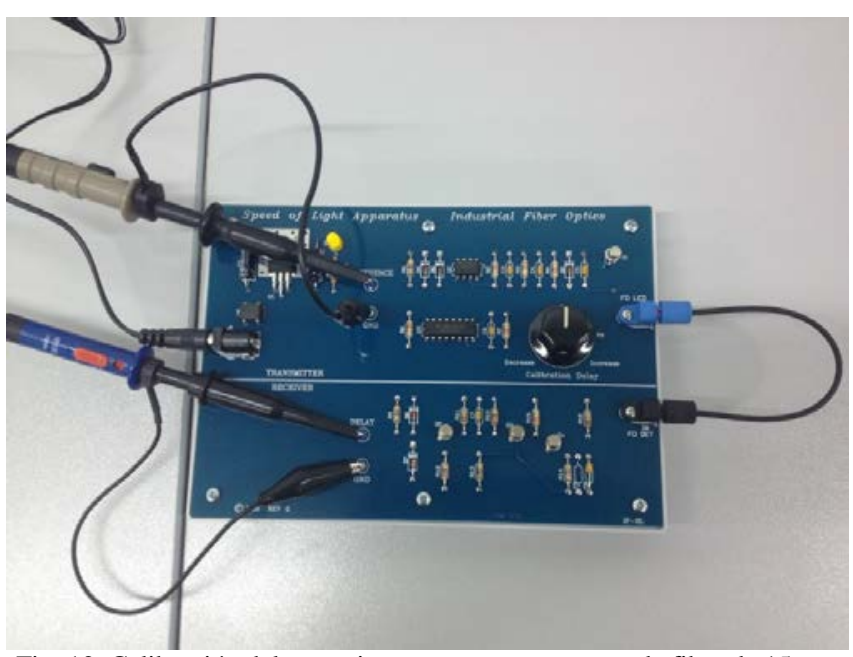

Fig. 12. Calibración del montaje con un pequeño tramo de fibra de $15 \mathrm{~cm}$.

Para ello, tras visualizar en el osciloscopio que el pulso emitido y el recibido, con el tramo de 15 $\mathrm{cm}$, presentaban cierto desfase temporal (Fig. 13), se utilizó el mando de Calibration Delay para situar ambos pulsos en el mismo instante de 


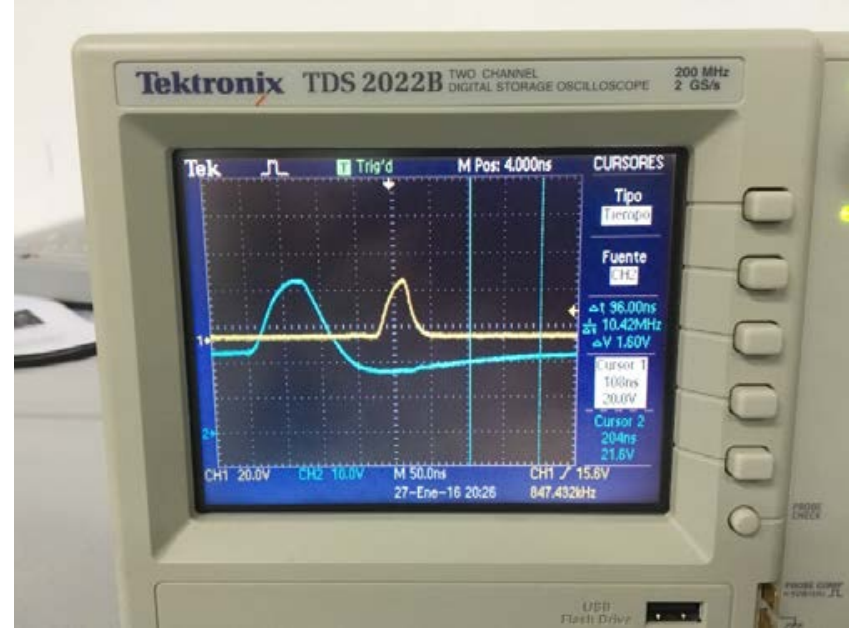

Fig. 13. Pantalla del osciloscopio en la que se observan el pulso emitido y el recibido (con el tramo de fibra de $15 \mathrm{~cm}$ ), presentando cierto desfase temporal.

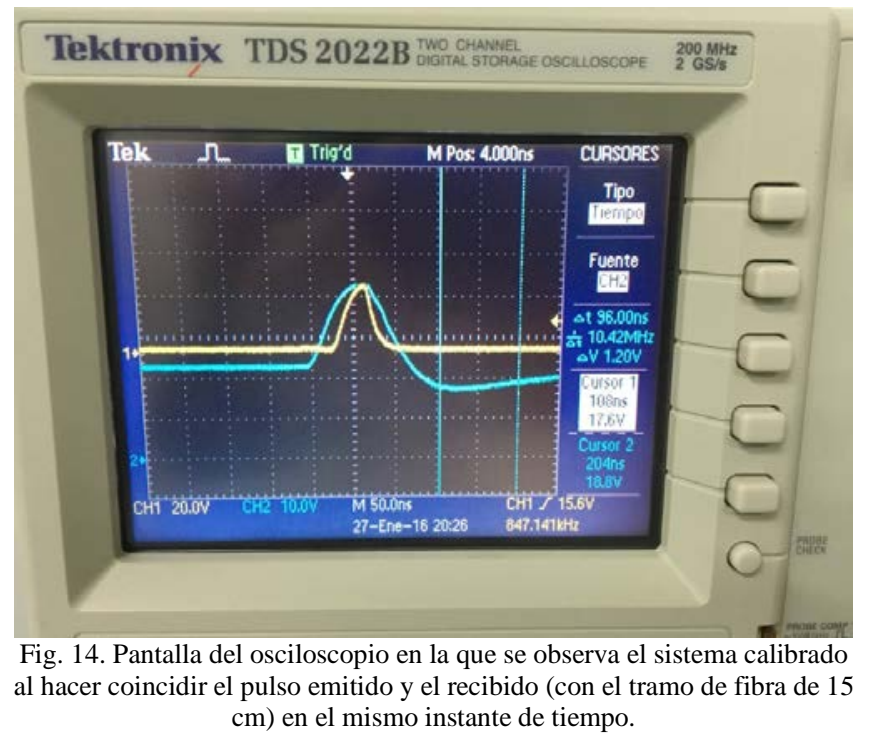

tiempo (Fig. 14), y así dar el montaje por calibrado.

A continuación, se sustituyó el tramo de fibra de $15 \mathrm{~cm}$ por el de $20 \mathrm{~m}$, tal y como se aprecia en la Fig. 10. De esta forma, tras emitir de nuevo un pulso de luz, se observaron en el osciloscopio el pulso emitido y el recibido (tras recorrer la distancia de $20 \mathrm{~m}$ a través del cristal del interior de la fibra óptica). Así, con ayuda de cursores que se situaron en el punto de máxima amplitud de cada uno de ellos, se pudieron extraer sus tiempos exactos de visualización (Fig. 15 y Fig. 16).

Concretamente, y tal y como puede apreciarse en las Fig. 15 y Fig. 16, el pulso de luz emitido se transmitió en un instante exacto correspondiente a 14 ns, y el pulso de luz recibido se detectó en un tiempo de 108 ns.

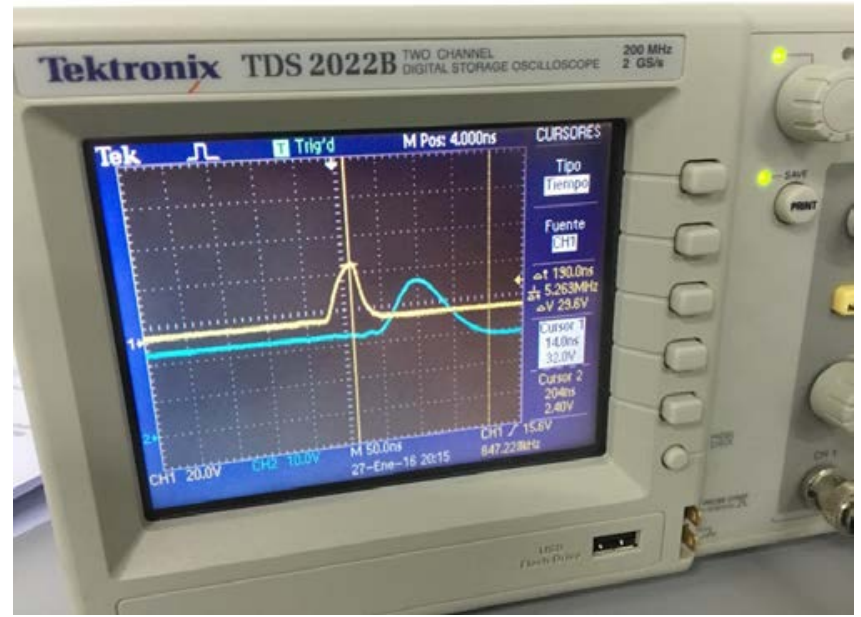

Fig. 15. Pantalla del osciloscopio en la que se observan el pulso de luz emitido y el recibido, con el cursor situado en el punto de máxima amplitud del emitido.

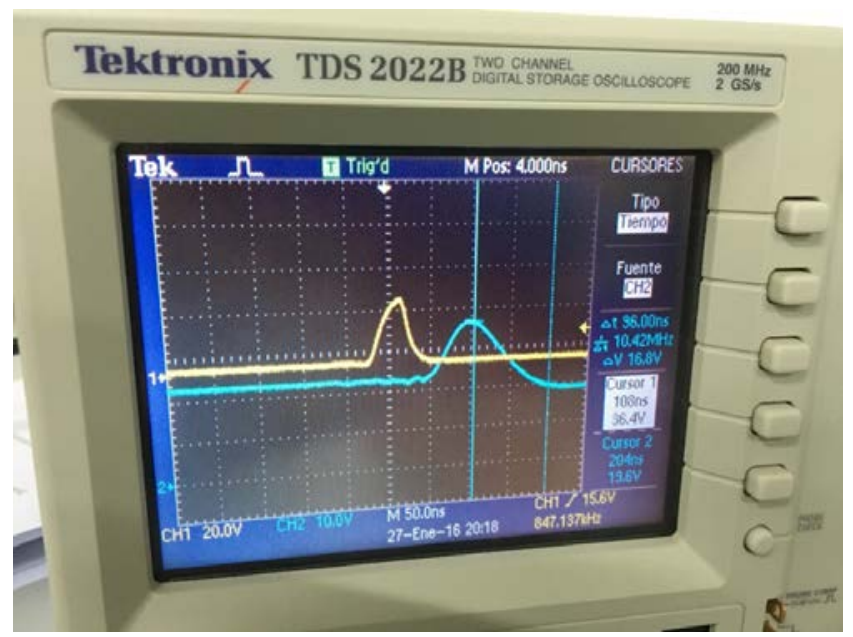

Fig. 16. Pantalla del osciloscopio en la que se observan el pulso de luz emitido y el recibido, con el cursor situado en el punto de máxima amplitud del recibido.

Por tanto, haciendo uso de la ecuación (4) ya indicada anteriormente, calculamos finalmente la velocidad de la luz en cristal como:

$$
\begin{gathered}
v_{\text {cristal }}=\frac{20}{108 \cdot 10^{-9}-14 \cdot 10^{-9}}= \\
\frac{20}{94 \cdot 10^{-9}}=2,127 \cdot 10^{8} \mathrm{~m} / \mathrm{s}=212700 \mathrm{~km} / \mathrm{s}
\end{gathered}
$$

Así mismo, utilizando la ecuación (3), se puede deducir el índice de refracción del cristal como:

$$
n_{\text {cristal }}=\frac{c}{v_{\text {cristal }}}=\frac{2,997 \cdot 10^{8}}{2,127 \cdot 10^{8}}=1,41
$$

que se aproxima bastante al valor conocido del interior de una fibra óptica (cristal de $\mathrm{SiO}_{2}$ ): 1,46. Concretamente, se ha obtenido con un error del $3,4 \%$. 
C. Medida de la velocidad de la luz en el agua

- Equipamiento necesario

- Láser.

- 2 porta-ángulos.

- Recipiente con agua.

- Líquido blanco que provoque scattering.

- Spray de humo o polvo de tiza.

- Calculadora.

\section{- Metodología}

En este caso, para obtener la velocidad de la luz en el agua, se optó por un método indirecto, en el que, primeramente, debemos hallar el índice de refracción del agua $\left(n_{\text {agua }}\right)$, para que, una vez deducido, podamos obtener la velocidad de la luz en este medio a través de la expresión:

$$
v_{\text {agua }}=\frac{c}{n_{\text {agua }}}
$$

En este sentido, con el fin de calcular el índice de refracción del agua, se consideró el siguiente montaje (Fig. 17):

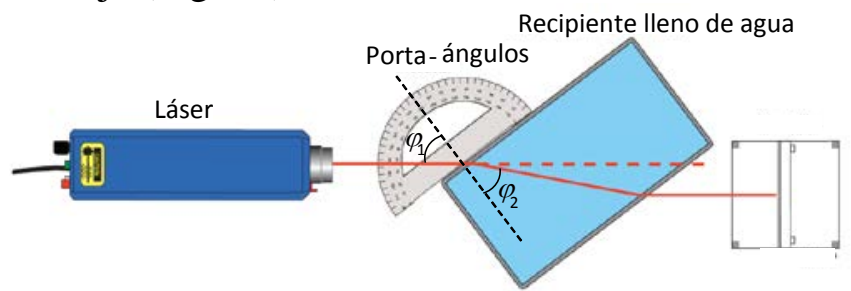

Fig. 17. Montaje para el experimento de medida del índice de refracción del agua. Fuente: http://www.i-fiberoptics.com/.

El método consiste en hacer incidir un rayo láser (emitido, en este caso, por un láser de HelioNeón de la empresa Industrial Fiber Optics-IF HN05) en un recipiente transparente con agua. Con el fin de que el rayo de luz sea visible mientras se propaga por el agua, a ésta se le añaden unas gotas de un líquido especial (una solución con partículas lo suficientemente pequeñas como para generar el fenómeno de scattering) y de color blanco (asumiremos que estas gotas de líquido apenas modifican el índice de refracción del agua). Para poder observar el rayo en el aire, se puede pulverizar por un momento con un spray de humo (también servirían partículas de tiza). Con ayuda de dos porta-ángulos, se miden tanto el ángulo del rayo incidente respecto a la perpendicular al borde del recipiente $\left(\varphi_{1}\right)$ como el ángulo que forma la luz respecto a dicha perpendicular una vez que se propaga dentro del agua $\left(\varphi_{2}\right)$, ya que el rayo se desviará respecto de la trayectoria que tenía antes de entrar en el recipiente (se refractará). Con estos datos, aplicamos la ley de Snell:

$$
n_{0} \operatorname{sen}\left(\varphi_{1}\right)=n_{\text {agua }} \operatorname{sen}\left(\varphi_{2}\right)
$$

donde $n_{0}$ es el índice de refracción del aire que, como ya se ha indicado anteriormente, es conocido y aproximaremos a 1 .

Por tanto:

$$
n_{\text {agua }}=\frac{\operatorname{sen}\left(\varphi_{1}\right)}{\operatorname{sen}\left(\varphi_{2}\right)}
$$

Con lo que la velocidad de la luz en el agua vendrá dada por la expresión (7).

- Resultados

En la Fig. 18, se observa el resultado que se obtuvo al hacer incidir el rayo láser sobre el recipiente lleno de agua.

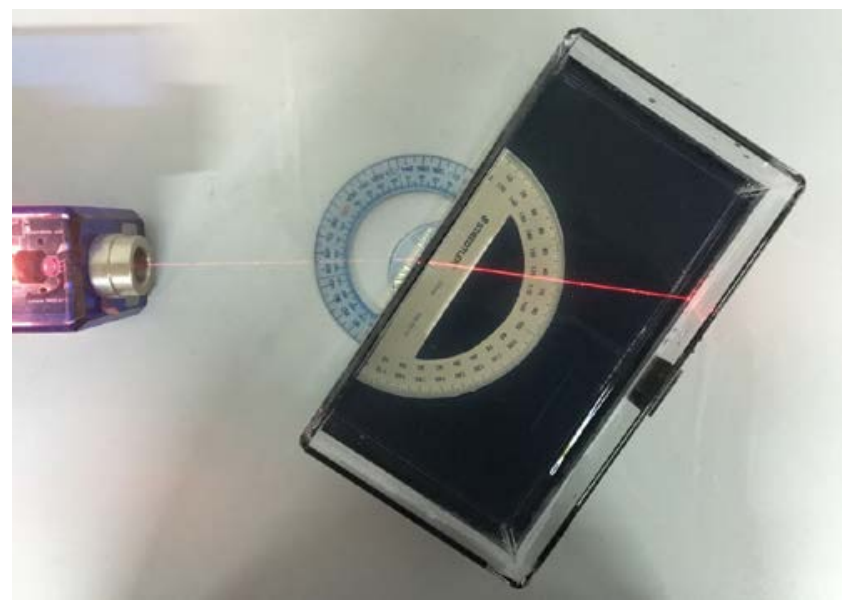

Fig. 18. Resultado del experimento para la medida del índice de refracción del agua.

Como se aprecia en la imagen, $\varphi_{1}=35^{\circ}$ y $\varphi_{2}=26^{\circ}$, por lo que:

$$
\begin{gathered}
\operatorname{sen}\left(35^{\circ}\right)=n_{\text {agua }} \operatorname{sen}\left(26^{\circ}\right) \\
n_{\text {agua }}=\frac{\operatorname{sen}\left(35^{\circ}\right)}{\operatorname{sen}\left(26^{\circ}\right)}=\frac{0,5735}{0,4383}=1,308
\end{gathered}
$$

que es un valor muy cercano al conocido de 1,33. Concretamente, se ha obtenido con un error del $1,65 \%$.

Y, finalmente: 


$$
\begin{gathered}
v_{\text {agua }}=\frac{2,99 \cdot 10^{8}}{1,308}= \\
2,28 \cdot 10^{8} \mathrm{~m} / \mathrm{s}=228000 \mathrm{~km} / \mathrm{s}
\end{gathered}
$$

\section{CONCLUSIONES}

Se han planteado una serie de propuestas para medir la velocidad de la luz en diferentes medios (aire, cristal y agua) dentro de un ámbito docente. Los experimentos pueden realizarse de manera relativamente sencilla dentro de cualquier laboratorio universitario y arrojan valores de la velocidad de la luz muy cercanos a los conocidos. De esta manera, se consigue que los estudiantes conozcan o afiancen, de una manera práctica, conceptos como la naturaleza de la luz o el índice de refracción de un medio. Además, las actividades de medición de la luz presentadas en este artículo suponen una manera divertida y práctica de enriquecer la enseñanza de la óptica y el electromagnetismo, familiarizarse con instrumentación específica como los osciloscopios o los emisores/receptores ópticos y entender la ciencia subyacente tras las fibras ópticas.

\section{REFERENCIAS}

[1] A. Pilot, A. M. W Bulte, Why do you "need to know"? Contextbased education. International Journal of Science Education, 28(9), 953-956. 2006.

[2] A. Pilot, A. M. W Bulte, The use of "contexts" as a challenge for the chemistry curriculum: Its successes and the need for further development and understanding. International Journal of Science Education, 28(9), 1087-1112. 2006.

[3] E. Pedrinaci Rodríguez, A. Caamaño-Ros, P. Cañal de León y A. de Pro Bueno, 11 Ideas Clave. El desarrollo de la competencia científica. Ed. Grao. 2012.

[4] J.A. Biretta, J. E. Lang. A simple velocity of light experiment, Am. J. Phys. 46(11), 1189. 1978.

[5] J. Cooke, M Martin, H. McCartney, B. Wilf. Direct determination of the speed of light as a general physics laboratory experiment. Am. J. Phys. 36, 847. 1968.

[6] C. E. Tyler. A pedagogical measurement of the velocity of light. Am. J. Phys. 49, 740-745. 1969.

[7] J. Vanderkooy, M. J.Beccario. An inexpensive, accurate laboratory determination of the velocity of light. Am. J. Phys. 41, pp. 272-275. 1973.

[8] W. Parker y A. Gold. Laboratory Measurement of the Velocity of Light. Am. J. Phys. 26, 481. 1958.

[9] J. Carlson, Speed of light measurement with the laser pointer. Phys. Teach. 34, 176-177. 1996.

[10] S. Mak, y D. Yip. The measurement of the speed of light using a laser pointer. Phys. Educ. 35(2) 95-100. 2000.

[11] K. Aoki, T. Mitsui. A tabletop experiment for the direct measurement of the speed of light. Am. J. Phys. 76, 812. 2008.

[12] E. Gülmez. Measuring the speed of light with a fiber optic kit: An undergraduate experiment. Am. J. Phys. 65, 614. 1997.

[13] J. Brody, L. Griffin, y P. Segre. Measurements of the speed of light in water using Foucault's technique. Am. J. Phys. (78) 6, 650-653. 2010.

[14] I. Martí-Vidal, S. Jiménez-Monferrer, y C. Cruz-Molina. Emulando a Römer: Medida de la Velocidad de la Luz Cronometrando los Eclipses de Io. Revista Española de Física (24) 3, 48-51. 2010.
[15] E. A. Mantero Castañeda. Medición de la velocidad de la luz. Rehaciendo el Experimento de Römer. Trabajo Fin de Grado. Universidad de la Laguna. 2016.

[16] O. Rømer. Philosophical Transactions of the Royal Society, 136, 893. 1677.

[17] A. R. Toledo Baca, y P. López Varo. Obtención de la velocidad de la luz con un horno microondas. Revista Española de Física, 25(2), 50-53. 2011.

[18] A. A. Michelson. Experimental Determination of the Velocity of Light. Proceedings of the American Association for the Advancement of Science. 71-77. 1879.

[19] R. J. Mackay y R.W. Oldford. Statistical Science, 15, 3, 254. 2000.

[20] M. A. Finocchiaro. Retrying Galileo, 1633-1992. University of California Press. Chap. 3, p.63. 2007.

[21] NIST. Fundamental physical constants. NIST Constants, Units, and Uncertainty. 2014.

[22] H. L. Fizeau. Sur une experience relative a la vitesse de propogation de la lumiere. Comptes Rendus. 1849.

[23] L. Foucault. Méthode générale pour mesurer la vitesse de la lumière dans l'air et les milieux transparents. Vitesses relatives de la lumière dans l'air et dans l'eau. Projet d'expérience sur la vitesse de propagation du calorique rayonnant. C. R. Hebd. Seances Acad. Sci. 30, 551-560. 1850. 